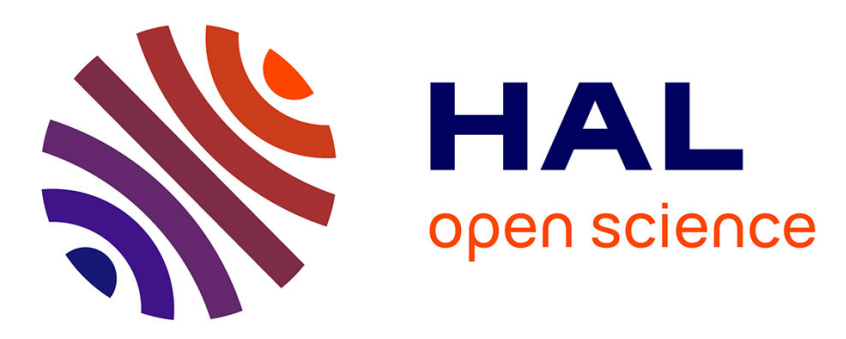

\title{
Nanometer Precision With a Planar Parallel Continuum Robot
}

Benjamin Mauze, Redwan Dahmouche, Guillaume Laurent, Antoine Andre, Patrick Rougeot, Patrick Sandoz, Cédric Clevy

\section{To cite this version:}

Benjamin Mauze, Redwan Dahmouche, Guillaume Laurent, Antoine Andre, Patrick Rougeot, et al.. Nanometer Precision With a Planar Parallel Continuum Robot. IEEE Robotics and Automation Letters, 2020, 5 (3), pp.3806- 3813. 10.1109/LRA.2020.2982360 . hal-02991169

\section{HAL Id: hal-02991169 \\ https://hal.science/hal-02991169}

Submitted on 5 Nov 2020

HAL is a multi-disciplinary open access archive for the deposit and dissemination of scientific research documents, whether they are published or not. The documents may come from teaching and research institutions in France or abroad, or from public or private research centers.
L'archive ouverte pluridisciplinaire HAL, est destinée au dépôt et à la diffusion de documents scientifiques de niveau recherche, publiés ou non, émanant des établissements d'enseignement et de recherche français ou étrangers, des laboratoires publics ou privés. 


\title{
Nanometer Precision with a Planar Parallel Continuum Robot
}

\author{
Benjamin Mauzé, Redwan Dahmouche, Guillaume J. Laurent, Antoine N. André, \\ Patrick Rougeot, Patrick Sandoz, Cédric Clévy
}

\begin{abstract}
In many cases, soft and continuum robots represent an interesting alternative to articulated robots because they have the advantages of miniaturization capability, safer interactions with humans and often simpler fabricating and integration. However, these benefits are usually considered to arise at the expense of accuracy and precision because of the soft or flexible limbs. This paper demonstrates that, with a proper design, a planar parallel continuum robot is capable of great precision. Indeed, the proposed 3-Degrees-of-Freedom planar parallel continuum robot exhibits a precision of $9.13 \mathrm{~nm}$ in position and $1.2 \mu \mathrm{rad}$ in orientation. In addition, the novel robotic design leverages the effect of the actuators' defects, making the robot more precise than its own actuators. Finally, the workspace of the proposed robot $\left(62.3 \mathrm{~mm}^{2}, 0.6452 \mathrm{rad}\right)$ is significantly larger than most compliant mechanisms, which is particularly interesting when both very high precision and relatively large displacements are required.
\end{abstract}

Index Terms-Soft Robot Applications; Micro/Nano Robots; Parallel Robots

\section{INTRODUCTION}

A FTER decades of development, industrial robots use today very mature technologies. Yet, they use bearings and gearboxes that limit their precision and accuracy due to backlashes, flexibilities and frictions. Parallel robots leverage some of these drawbacks thanks to more rigid structures but still basically experience the same types of defects [1].

In applications such as micro and nano assembly, automated biological cell manipulation, X-ray lithography, and others, where high-grade precision positioning is required, such mechanical components should be avoided [2], [3]. Flexure hinge-based compliant structures actuated by piezoelectric, electromagnetic, electrostatic or electrothermal actuators are thus preferred [4]-[7]. However, this type of mechanism has very limited workspace in position as well as in rotation [5].

Soft and continuum robots have interesting properties over articulated and flexure-based robots. Thanks to innovative

Manuscript received: October, 15, Year; Revised February, 6, 2020; Accepted March, 4, 2020.

This paper was recommended for publication by Editor C.Laschi upon evaluation of the Associate Editor and Reviewers' comments.

This work was supported by ISITE-BFC (contract ANR-15-IDEX-03), Equipex ROBOTEX (ANR-10-EQPX-44-01), ANR (ANR-19-CE10-000401), by Région Bourgogne Franche-Comté and the EIPHI Graduate School (ANR-17-EURE-0002).

The authors are with FEMTO-ST Institute, Univ. Bourgogne FrancheComté, UMR CNRS 6174, Besançon, France benjamin.mauze, redwan.dahmouche, guillaume.laurent, antoine.andre, patrick.rougeot, patrick.sandoz, cedric.clevylfemto-st.fr

Digital Object Identifier (DOI): see top of this page. designs [8], [9], actuation systems [10] and materials [11], classical robots have seen improved in miniaturization capability [12], [13], lightweight designs, adaptability [14], payloadto-robot weight ratios [15], length-to-width ratios [16] and others characteristics [17].

However, a property of soft and continuum robots that is currently missing is precision. Indeed, very little work has been done in this field. For instance, one of the rare studies found a precision of about $1 \mathrm{~mm}$ for a serial continuum robot [18]. In addition, Orekhov et al. [19] proposed a surgical Parallel Continuum Manipulator with a large workspace and a precision of $0.88 \mathrm{~mm}$ in position and $1.96 \mathrm{deg}$ in rotation. Black et al. [20] studied the pros and cons of Parallel Continuum Robots (PCR) and pointed out that an analysis of their performance was still needed.

Atuzarra et al. [21], by analyzing the characteristics of PCR, announced that they may achieve better precision than their serial counterparts. However, no evaluation was made of their potential precision compared to other technologies such as classical parallel robots. The current conjecture, from the industrial and academic practices, leads to think that articulated robots are better suitable for precision manipulation [22].

The question that arises then is, "Is it possible to combine the advantages of the different technologies to obtain a high precision over a large workspace?"

In this paper, simulated and experimental results show that parallel continuum robots are relevant candidates for high precision manipulation. Indeed, their mechanical structure does not have mechanical joints. Moreover, the limbs' bending can be large, with appropriate materials allowing relatively large displacement range compared to compliant mechanisms. This paper shows that a 3-Degrees-of-Freedom (3-DoF) planar parallel continuum robot can reach nanometer precision along a relatively large workspace, ranking it among the best solutions for high-grade precision applications.

The next section introduces the 3-DoF PCR structure whose model is provided in Section III. The design and prototype development is proposed in Section IV. Precision performances of the robot are investigated first by simulation in Section $\mathrm{V}$ and then experimentally in section VI.

\section{3-DoF PARALlel CONTINUUm RoBot}

This paper aims to demonstrate that parallel continuum robots (PCR) can achieve high positioning precision (comparable with flexure hinge-based compliant stages) while having 
longer travel ranges. For that purpose, a planar PCR, inspired by the well-known 3-PRR parallel robot, was designed.

The regular 3-PRR mechanism [23] is composed of three planar kinematic chains, each with a prismatic joint and two revolute joints, all connecting the fixed base to the moving platform. The direction of the three chains are star-shaped with 120-degree angles. This mechanism allows positioning of the platform along three-degrees-of-freedom, $x, y$, and $\theta$ in the plane according to the prismatic joint values $q_{1}, q_{2}$, and $q_{3}$.

The proposed design of the planar PCR is also composed of three kinematic chains but links and joints are replaced by slender rods that provide large and continuous deformations. Each rod is connected to a precision linear actuator and to the rigid moving platform. The motion of the platform is restricted to the 3-DoF planar displacements.

The rods are clamped on their proximal ends, denoted $A_{i}$, to the linear stages in line with the direction of their motions. The three stages are arranged at 120-degree angles such that $A_{1} A_{2} A_{3}$ is an equilateral triangle when the actuators are in their reference positions. The clamping points of the distal ends of the rods, $B_{i}$, to the mobile platform also form an equilateral triangle with vertices $B_{1}, B_{2}$ and $B_{3}$.

As illustrated in Fig. 1, only four parameters are required to describe the manipulator geometry:

- $l$ is the length at the free-stress configuration of the rods (the rods are straight before assembling);

- $r_{P}$ is the radius of the circle defined by the three distal ends of the rods $B_{1}, B_{2}, B_{3}$;

- $r_{A}$ is the radius of the circle defined by the three proximal ends $A_{1}, A_{2}, A_{3}$ of the rods when the actuators are in their reference positions;

- $\alpha$ corresponds to the angle between the prismatic joint axis and the direction of the center of the circle (for $\alpha=$ 0 , the actuator line passes through the center of the circle; for $\alpha=\pi / 2$, the actuator line is tangent to the circle).

\section{MODELING}

In this section, we detail the model of the proposed PCR structure that will be used to simulate its precision.

\section{A. Frames and Transformations}

For the purpose of analysis, we attach a work reference frame $\left(O_{W}, \mathbf{x}_{W}, \mathbf{y}_{W}, \mathbf{z}_{W}\right)$ to the fixed base with its origin located at the center of the circumscribed circle of triangle $A_{1} A_{2} A_{3}$ when the actuators are in their reference positions and with the $\mathbf{z}_{W}$ axis perpendicular to the base. A mobile frame $\left(O_{P}, \mathbf{x}_{P}, \mathbf{y}_{P}, \mathbf{z}_{P}\right)$ is attached to the moving platform with its origin located at the center $\mathrm{G}$ of the triangle $B_{1} B_{2} B_{3}$. Finally, mobile frames are attached to the proximal ends and to the distal ends of each rod (Fig. 1).

These frames allow us to define the following transformations:

- The transformations ${ }^{W} \mathbf{T}_{A_{i}}$ from the work frame to the rod's proximal end, which are each a function of the prismatic articulate variable $q_{i}$ each;

- The rod transformations ${ }^{A_{i}} \mathbf{T}_{B_{i}}$ that define the relative position and orientation between the proximal and the

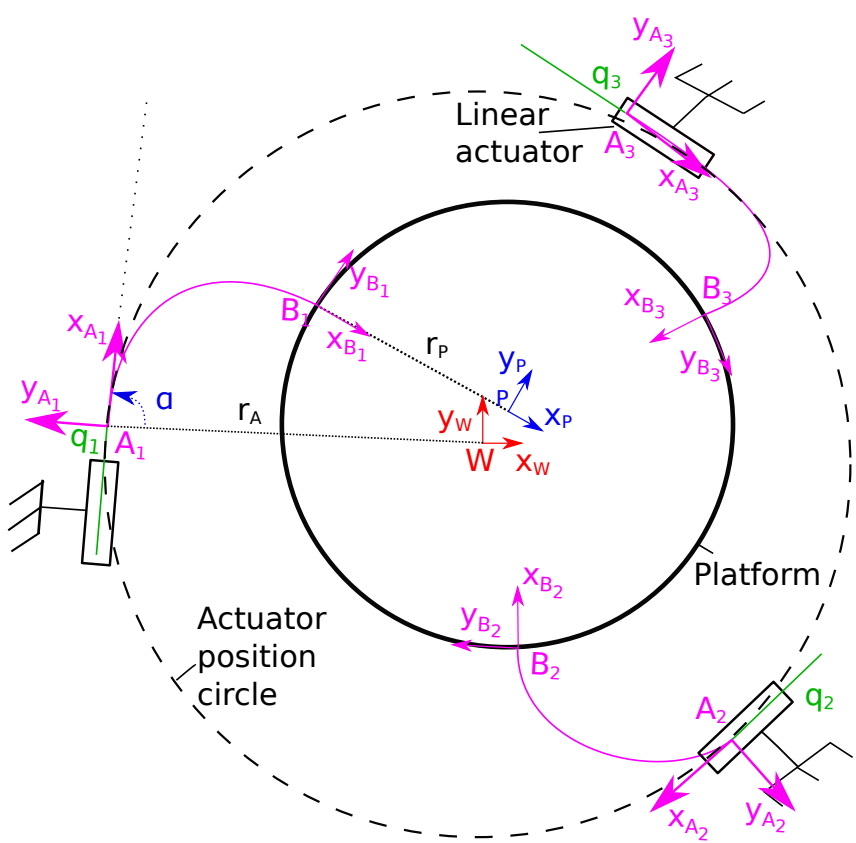

Fig. 1: Model of the new planar Parallel Continuum Robot.

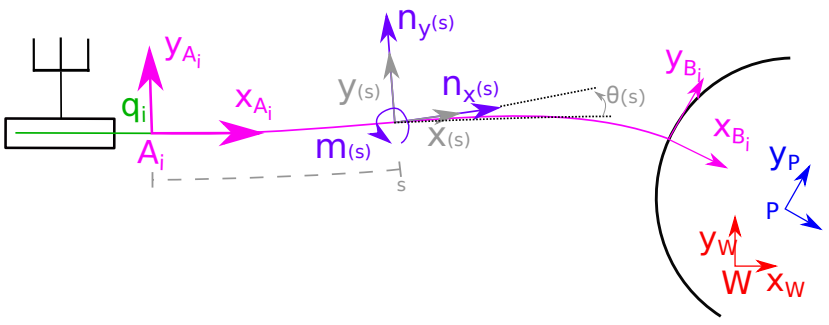

Fig. 2: Rod model within the $(O, \mathbf{x}, \mathbf{y})$ plane with frames and boundary conditions

distal end of each rod, depending on the external forces and moments applied to it;

- The transformations ${ }^{B_{i}} \mathbf{T}_{P}$ from each rod's distal end to the mobile frame, which are constant since the platform is rigid;

- The transformation ${ }^{W} \mathbf{T}_{P}$ that gives the position and the orientation of the mobile platform with regards to the work frame.

\section{B. Rods Modeling}

1) Kirchhoff Rod Equations: The rods are slender elements, straight in their free-stress configuration. However, to avoid singular configurations, the rods are constrained in the home configuration (see Fig. 2). The characteristic dimension of their cross-section (diameter for cylindrical rod) is more than 100 times smaller than the rod's length. Thus, shear and elongation can be neglected. Considering those assumptions, the Cosserat rod model can be simplified to the Kirchhoff model.

In this article, we consider the planar case of this model. We suppose that the movement of each rod is planar inside the $(O, \mathbf{x}, \mathbf{y})$ plane. All variables are illustrated in Fig. 2. 
A scalar parameter $s$ represents the curvilinear abscissa over the entire rod, $s \in[0, l]$ where $l$ is the free-stress length of a rod.

The shape of a rod along its arc length is defined by the cross-section centroid position $\mathbf{p}(s)=\left[\begin{array}{l}x(s) \\ y(s)\end{array}\right]$ represented in the frame attached to its proximal end $A_{i}$ and $\mathbf{R}(s)=$ $\left[\begin{array}{cc}\cos \theta(s) & -\sin \theta(s) \\ \sin \theta(s) & \cos \theta(s)\end{array}\right]$ the rotation matrix between the frame attached to $A_{i}$ and the one attached to $\mathbf{p}(s)$.

The evolution of position $\mathbf{v}(s) \in \mathbb{R}^{2}$ and orientation $u_{z}(s) \in \mathbb{R}$ along the arc length, related to the material strain, can be expressed in the local frame as:

$$
\begin{aligned}
\mathbf{R}(s)^{T} \frac{d \mathbf{p}(s)}{d s} & =\mathbf{v}(s) \\
\mathbf{R}(s)^{T} \frac{d \mathbf{R}(s)}{d s} & =\left[\begin{array}{cc}
0 & -u_{z}(s) \\
u_{z}(s) & 0
\end{array}\right]
\end{aligned}
$$

The internal force $\mathbf{n}(s)=\left[\begin{array}{l}n_{x}(s) \\ n_{y}(s)\end{array}\right]$ and moment $m(s)$ are defined with respect to the arc length. We assume that no distributed external forces or moments are applied on the rod. The nonlinear equations of the static equilibrium of Kirchhoff rod yield:

$$
\begin{gathered}
\frac{d \mathbf{n}(s)}{d s}=0 \\
\frac{d m(s)}{d s}+\frac{d \mathbf{p}(s)}{d s} \times \mathbf{n}(s)=0
\end{gathered}
$$

From all previously defined equations, the system of differential equations is the following:

$$
\left\{\begin{array}{c}
\frac{d x(s)}{d s} \\
\frac{d y(s)}{d s} \\
\frac{d \theta(s)}{d s} \\
\frac{d n_{x}(s)}{d s} \\
\frac{d n_{y}(s)}{d s} \\
\frac{d m(s)}{d s}
\end{array}\right\}=\left\{\begin{array}{c}
\cos (\theta(s)) \\
\sin (\theta(s)) \\
\frac{m}{E I}+u_{z}(0) \\
0 \\
0 \\
n_{x} \sin (\theta(s))-n_{y} \cos (\theta(s))
\end{array}\right\}
$$

Where $E$ is the Young modulus of the rod material, $u_{z}(0)$ is the initial orientation of the rod, which is zero in our case, and $I$ is the second area moment of the rod cross-section.

This system of equations describes the shape of the rod and the internal forces and moments. The rod shape corresponds to the transformation between the point $A_{i}$ and $B_{i}$ where $i \in$ $\{1,2,3\}$ is the limb number.

2) Proximal Boundary Conditions: The proximal boundary condition of the $i$ th rod corresponds to the position of the point $A_{i}$. The transformation ${ }^{W} \mathbf{T}_{A_{i}}$ from the work frame to the rod's proximal end depends on the joint coordinate $q_{i}$. When $q_{i}=0$, the joint is in the reference position. In this paper, the considered joint is prismatic, thus the transformation ${ }^{W} \mathbf{T}_{A_{i}}$ follows a translation in the direction of the joint. One can notice that this transformation can be calculated for other kinds of actuators.

3) Distal Boundary Conditions: The distal ends of the rods are coupled through their connection with the platform. Rigidbody conditions between the distal ends of the rods and the platform are the following:

$$
{ }^{W} \mathbf{T}_{B_{i}}={ }^{W} \mathbf{T}_{P} \cdot{ }^{P} \mathbf{T}_{B_{i}}
$$



Fig. 3: Prototype of the proposed Parallel Continuum Robot.

Using both boundaries conditions, the $i^{t h}$ rod transformation $A_{i} \mathbf{T}_{B_{i}}$ is then given by:

$$
{ }^{A_{i}} \mathbf{T}_{B_{i}}={ }^{A_{i}} \mathbf{T}_{W} \cdot{ }^{W} \mathbf{T}_{B_{i}}
$$

$\mathrm{e}$

4) Static Equilibrium Conditions: The static equilibrium conditions of the platform, needed in order to determine the position and orientation of the platform, yield:

$$
\begin{array}{r}
\sum_{i=1}^{3}\left[\mathbf{n}_{i}(L)\right]-\mathbf{f}_{P}=0 \\
\sum_{i=1}^{3}\left[\mathbf{p}_{B_{i}} \times \mathbf{n}_{i}(L)+m_{i}\right]-\mathbf{p}_{P} \times \mathbf{f}_{P}-m_{P}=0
\end{array}
$$

where $\mathbf{p}_{B_{i}}$ and $\mathbf{p}_{P}$ are the positions in the work frame of $B_{i}$ and $P, \mathbf{f}_{P}$ and $m_{P}$ are respectively the external forces and moment applied on the platform and $\mathbf{n}_{\mathbf{i}}$ and $m_{i}$ the ones applied by the rods expressed in the work frame.

\section{PCR DESIGN}

This section details the different parts of the PCR and its assembly. Fig. 3 shows the developed prototype.

The mobile platform is a silicon wafer of radius $r_{P}=51.85 \mathrm{~mm}$ ( 2 inches) on which three fiber holders were glued 120 degrees apart. This choice of platform was motivated by a potential application of wafer positioning.

Underneath the platform, a $50 \mathrm{~mm}$ diameter vacuum preloaded air-bearing S205001, from the IBS company, maintains the platforms at a stable height $( \pm 5 \mu \mathrm{m})$. The air-bearing is mounted on a manual linear stage, Newport M-DS25-Z, which allows the level of the wafer to be adjusted.

Fiber holders are aluminum pieces drilled on one of their side in order to insert the flexible rods. The rods are $125 \mu \mathrm{m}$ diameter single-core optical fibers, which were stripped of the plastic part. Their Young modulus E is $73 \mathrm{GPa}$. Their length at their free-stress configuration is $30 \mathrm{~mm}$. They are clamped in line with the translation axis of the actuator.

The robot limbs are actuated by stick-slip piezo-electric linear actuators SmarAct slc-1730-s-hv whose resolution is about $1 \mathrm{~nm}$. Their precision in closed-loop is $18.02 \mathrm{~nm}$ for a displacement of $100 \mu \mathrm{m}$. Those actuators are a good 
TABLE I: Unknowns, inputs and outputs for solving the two kinematic models. The index $i$ is the number of the limbs which is equal to 1,2 and 3.

\begin{tabular}{cccc}
\hline Kinematic Model & Unknowns & Input & Output \\
\hline FKM & $\mathbf{n}_{\mathbf{i}}, m_{i}, \mathbf{p}_{P}, \theta_{P}$ & $q_{i}$ & $\mathbf{p}_{P}, \theta_{P}$ \\
\hline IKM & $\mathbf{n}_{\mathbf{i}}, m_{i}, q_{i}$ & $\mathbf{p}_{P}, \theta_{P}$ & $q_{i}$ \\
\hline
\end{tabular}

trade-off between size, travel range and resolution. Moreover, their compatibility with high vacuum opens the possibility to integrate them into a scanning electron microscope thanks to a more compact design in which the air-bearing would not be required.

Below the actuators, a manual rotation stage Newport MRS40 allows setting the angle $\alpha$ at 15 degrees. Below those two elements, two manual linear translation stages, Newport M-SDS-40, allow setting the distance $r_{A}$ at $85 \mathrm{~mm}$. Those manual stages allow the assembly of the prototype. They adjust the initial actuators' position to fix the corresponding holders, reduce the initial stresses inside the fiber and, with the manual stage underneath the air-bearing, adjust the planarity of the robot.

The footprint of the prototype is less than an equilateral triangle with side of $235 \mathrm{~mm}$.

\section{Simulated Precision Evaluation}

In this section, the simulated precision along the workspace of the PCR prototype is evaluated by Monte Carlo simulations.

\section{A. Software Implementation}

The quasi-static behavior of the previously defined PCR is simulated using two algorithms. Fig. $4 \mathrm{a}$ and Fig. $4 \mathrm{~b}$ represent respectively the two routines used to solve the two kinematic models, the Forward Kinematic Model (FKM) and the Inverse Kinematic Model (IKM). Both models are numerically solved with a shooting method.

Table I points out the unknowns, inputs and outputs for both algorithms. $\mathbf{p}_{P}=\left[\begin{array}{l}x_{P} \\ y_{P}\end{array}\right]$ and $\theta_{P}$ correspond respectively to the position and the orientation of the mobile platform in the work frame. We use the fourth-order Runge-Kutta method in order to integrate spatially the system of equations. The optimization process is performed using the built-in function lsqnonlin from Matlab software. The residue is the vector composed of the static equilibrium equations (6) and the rigidbody conditions (4) between the platform and the distal end of the rod.

The gravity is compensated by the air-bearing so we assume that no external forces or moments are applied to the platform when the platform is in equilibrium. By considering as null the external forces and moments, respectively $\mathbf{f}_{P}$ and $m_{P}$, the equations (3) and (6) are simplified.

\section{B. Workspace Simulation}

The flexibility of the PCR's elements allows them to have a relatively large workspace. In order to demonstrate the (a)



(b)



Fig. 4: Flowchart of the shooting method used to numerically solve the boundary conditions problem for (a) the Forward Kinematic Model (FKM) and (b) the Inverse Kinematic Model (IKM). For both cases, the spatial integration uses the fourthorder Runge-Kutta (RK4) method. "Res." in the flowchart represents the optimization residues.

precision of the proposed structure along its motion range without breaking the limbs, the safe workspace must be estimated by simulation first.

The workspace simulation is performed using the IKM (Fig. $4 \mathrm{~b})$. It has been computed following the method proposed by Merlet et al. [24]. Stable and reachable positions are computed using the IKM considering fixed platform's orientations. The position workspace for the initial platform's orientation is described and illustrated in Fig.5.

At each position, the stress inside the legs is computed and the maximal value is reported. The maximal stress variations inside the robot's legs are illustrated by the color of the 


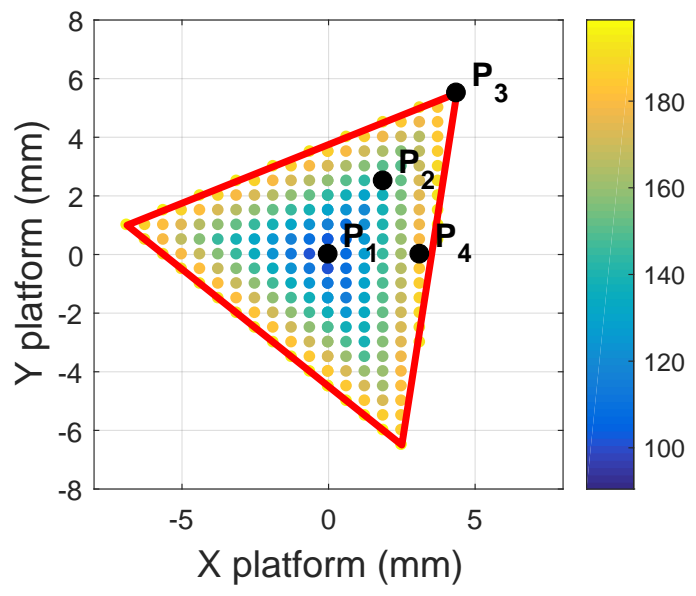

Fig. 5: Simulated workspace in position for a constant orientation of the platform (initial orientation) and for maximal stresses in the limbs below $200 \mathrm{MPa}$. The workspace is sampled by steps of $250 \mu \mathrm{m}$. The colors correspond to the maximal values of the stresses inside the three legs. The four black points $P_{1}, P_{2}, P_{3}$ and $P_{4}$ are the positions where the precision will be quantified.

corresponding position. To avoid breaking the robot's limbs, the workspace is limited to the area in which the maximal stress is below $200 \mathrm{MPa}$. This value was chosen by considering the safest elastic limit of the optic fiber, which varies between $200 \mathrm{MPa}$ and $700 \mathrm{MPa}$ for standard optical fiber without the acrilate protective coating [25].

The area of this safe workspace is equal to $62.3 \mathrm{~mm}^{2}$. Despite the fact that this workspace does not exploit the full actuators stroke, it is far larger than the best XY $\theta$ flexure hinge-based compliant mechanisms reported $\left(16 \mathrm{~mm}^{2}\right)$ [6]. Fig.5 shows the safe workspace and the four points $P_{1}, P_{2}$, $P_{3}$ and $P_{4}$ that were considered to quantify the precision of the robot in different positions. Due to the symmetries of the structure, these four points seem to be representative of the precision over the robot's safe workspace.

\section{Monte-Carlo Evaluation of the Precision}

1) Precision Definition: The precision of the robot is defined by its position and orientation precision. The position precision is defined as the standard deviation of the distance to the mean position of the platform, $s t d_{l}$.

$$
\begin{aligned}
\bar{l} & =\frac{1}{n} \sum_{j=1}^{n} l_{j} \\
l_{j} & =\sqrt{\left(x_{P_{j}}-\frac{1}{n} \sum_{i=1}^{n} x_{P_{i}}\right)^{2}+\left(y_{P_{j}}-\frac{1}{n} \sum_{i=1}^{n} y_{P_{i}}\right)^{2}} \\
s t d_{l} & =\sqrt{\frac{\sum_{j=1}^{n}\left(l_{j}-\bar{l}\right)^{2}}{n-1}}
\end{aligned}
$$

(a)
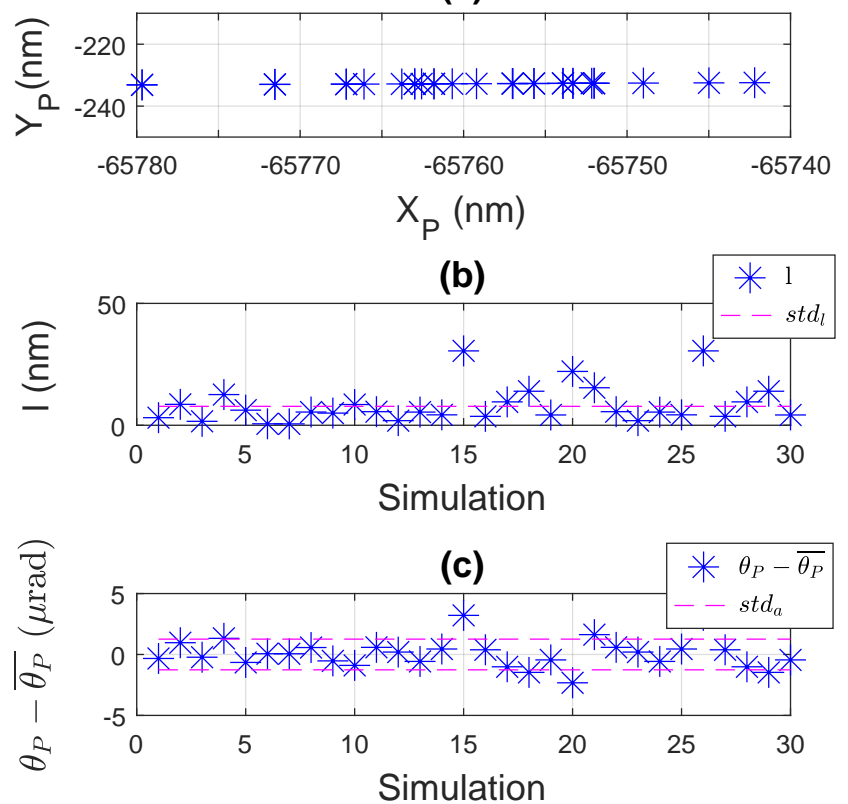

Fig. 6: Results of the propagation of uncertainty from the actuators to the robot's end-effector based on Monte Carlo simulations. The considered displacement is a translation of $100 \mu \mathrm{m}$ along $q_{1}$ from its initial position. (a) Platform coordinates in the work frame. (b) Distance deviation (distances to the mean position). (c) Angle deviation (differences between angles and their mean).

The orientation precision is defined as the standard deviation std $_{a}$ with :

$$
s t d_{a}=\sqrt{\frac{\sum_{j=1}^{n}\left(\theta_{P j}-\frac{1}{n} \sum_{i=1}^{n} \theta_{P i}\right)^{2}}{n-1}}
$$

Using the previously defined model and algorithms, the simulated precision of the robot is quantified by taking into account the actuation uncertainty.

2) Monte Carlo Method: This precision is simulated using the Monte Carlo method for the propagation of uncertainty distributions. This method was introduced by the Joint Committee for Guides in Metrology (JCGM) [26].

One considers the uncertainties of translation in the direction of the actuator displacement. Those uncertainties follow a normal distribution with a standard deviation of $18.02 \mathrm{~nm}$ for a displacement of $100 \mu \mathrm{m}$. This value was extracted from the manufacturer's documents.

The principle of the method is the following. One considered $n$ values of $q_{1}$ sorting from a normal distribution with a mean value is $100 \mu \mathrm{m}$ and a standard deviation of $18.02 \mathrm{~nm}$, which reflect respectively the desired position and precision of the actuator. Those joint coordinates are considered as inputs to the FKM to calculate the position and the orientation of the platform as outputs. The value $n$ was set to 30 to be 
TABLE II: Simulated and experimental position and orientation precision for different considered cases. The first four cases correspond to the four points chosen in the workspace and the last case corresponds to the addition of the uncertainties of the three actuators at the initial position $\left(P_{1}\right)$.

\begin{tabular}{ccccc}
\hline & \multicolumn{2}{c}{ Position Precision } & \multicolumn{2}{c}{ Orientation Precision } \\
\cline { 2 - 5 } & Simulated & Experimental & Simulated & Experimental \\
\hline P1 & $7.77 \mathrm{~nm}$ & $9.13 \mathrm{~nm}$ & $1.26 \mu \mathrm{rad}$ & $1.2 \mu \mathrm{rad}$ \\
\hline P2 & $16.73 \mathrm{~nm}$ & $12.7 \mathrm{~nm}$ & $1.74 \mu \mathrm{rad}$ & $0.71 \mu \mathrm{rad}$ \\
\hline P3 & $29.87 \mathrm{~nm}$ & $19.95 \mathrm{~nm}$ & $1.89 \mu \mathrm{rad}$ & $1.48 \mu \mathrm{rad}$ \\
\hline P4 & $12.56 \mathrm{~nm}$ & $12.18 \mathrm{~nm}$ & $1.82 \mu \mathrm{rad}$ & $1.75 \mu \mathrm{rad}$ \\
\hline P1-3 act. & $9.5 \mathrm{~nm}$ & $12.86 \mathrm{~nm}$ & $1.77 \mu \mathrm{rad}$ & $1.36 \mu \mathrm{rad}$ \\
\hline
\end{tabular}

statistically representative. Using this method, the precision for the previously considered points were estimated by simulation.

3) Simulation Results: Fig. 6 presents the results of the propagation of uncertainty from the actuators to the platform considering the displacement along $q_{1}$ from its initial position (P1 in Fig. 5). Fig. 6(a) shows the platform position where we can see the direction of uncertainty. Fig. 6(b) shows the distance deviation to the mean position $\left(l_{j}\right.$ in equation (7)). The standard deviation of those distances corresponds to the simulated position precision of the robot. Fig. 6(c) shows the angle deviation.

The angle standard deviation corresponds to the simulated orientation precision.

Additional simulations have been performed for the chosen points and considering the uncertainties of the three actuators. The results of the simulations are reported in Table II. In the last case, a displacement of $100 \mu \mathrm{m}$ is simulated for each actuator moving together from the robot's initial position.

One can notice that the precision of the robot's platform is better than the actuators precision. One explanation for this result is that, depending on their configurations, most parallel robots kinematics exhibit the property of amplifying or reducing the actuators motions. In the last case, the robot kinematics may leverage the actuators positioning uncertainties. Although, the presented parallel continuum robots has an additional property in which the rods act like springs that absorb the actuators motions. Thus, a fraction of the actuators work is not transmitted to the platform as motion but stored into the rods as a potential energy (rods bending). This property, which can be found only in deformable robots, may contribute in enhancing the platform's precision against the actuators defects.

The simulated values obtained in this section were checked against the ones obtained experimentally.

\section{Vi. EXPerimental Precision Evaluation}

In this section, the experimental set-up is described and the robot's precision is quantified experimentally.

\section{A. Measurement system}

The measurement system can be seen in Fig. 7. All the components are placed on an anti-vibration table to reduce the vibrations transmitted through the ground. The motion of

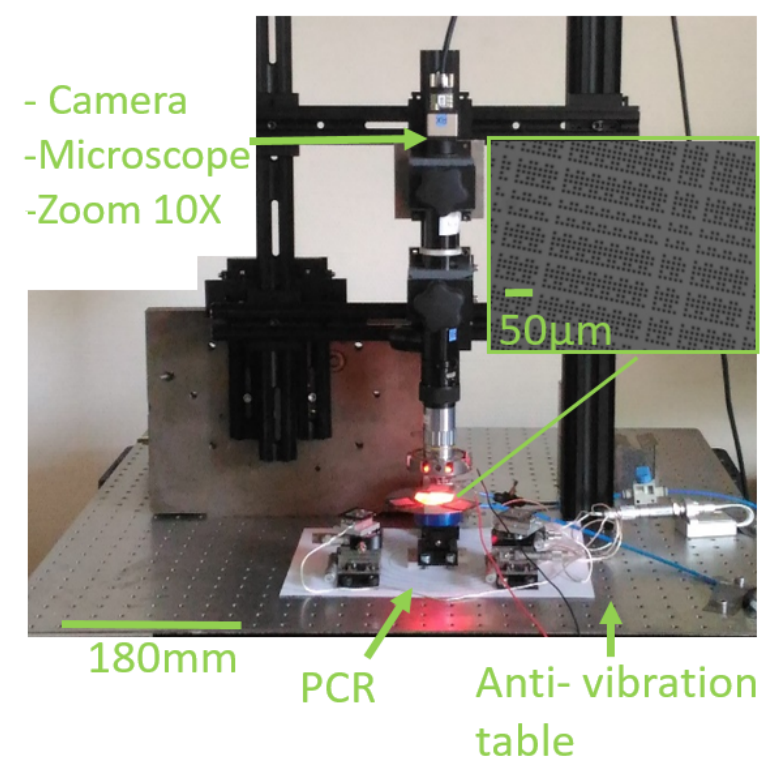

Fig. 7: Experimental setup showing the measurement system and the PCR prototype attached to an anti-vibration table. Pattern used with a Fast Fourier Transform phase-based algorithm. This algorithm provides position and orientation with a resolution of $0.5 \mathrm{~nm}$ and $1 \mu \mathrm{rad}$. [27]

the robot, shown in Fig. 3 and presented in section IV, is captured by a vision measurement system that is composed of a camera IDS UI3880CP-C-HR-R2, an Edmund microscope tube and a Mitutoyo $10 \times$ zoom. The camera is configured with an exposure time of $150 \mathrm{~ms}$ and takes 6.5 pictures per second of a calibrated pattern (Fig. 7) glued on the platform. All images are recorded after a warm-up cycle of the camera of at least one hour.

The pattern was manufactured by etching a chromium layer onto a transparent glass wafer. The periodicity between features is $9 \mu \mathrm{m}$ along the two directions. The pattern was fabricated by direct laser writing with an off-the-shelf instrument (Heidelberg DWL200) whose position and displacements are continuously controlled by a HeNe laser interferometer.

A phase-based method is used in order to get an accurate position and orientation measurement of the platform. It measures the phase of the image in the Fourier domains in order to get a sub-nanometric resolution. This algorithm together with a binary code provides the absolute pose of the pattern with a resolution of $0.5 \mathrm{~nm}$ in both directions and $1 \mu \mathrm{rad}$ for the orientation. More details on this measuring method can be found in [27]-[30].

\section{B. Preliminary Experiments}

A vision-based measurement system can be disturbed by environmental factors. Quantifying their impact enables defining a threshold below which the precision will not be distinguishable from the environment noise.

Three different cases are considered. The first one represents the quantification of the measurement noise, a.e. before powering the air-bearing or the actuators. The second one is the uncertainty change due to the air-bearing. The last 
(a)

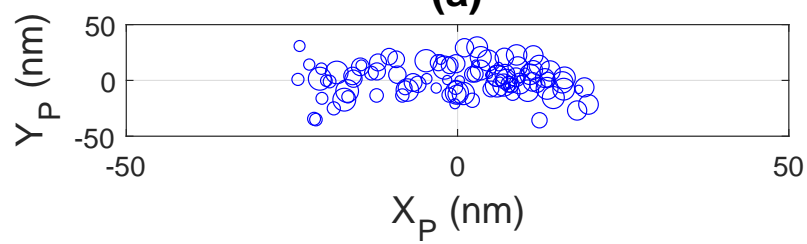

(b)

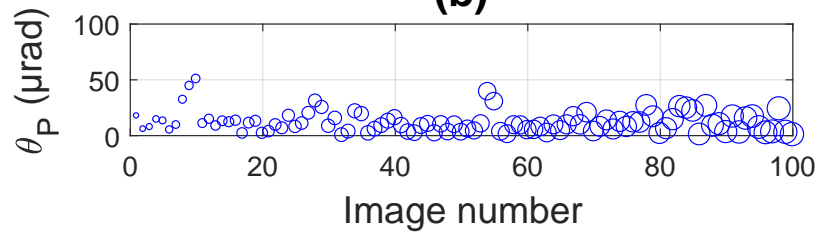

Fig. 8: (a) Quantification of the positioning measurement uncertainty and (b) the orientation measurement uncertainty when the robot is holding a position. The points' size depends on the image acquisition time.

one corresponds to the normal functioning conditions when the robot is holding a stable position, for instance, its initial position.

One hundred images are recorded for about one minute. The vision algorithm is used to obtain the position and the orientation of the platform. Distances to the mean $\left(l_{j}\right.$ in equation (7)) and the differences between angles and their mean are computed. The standard deviations of those values quantify the measurement uncertainty threshold.

For the three cases detailed above, the uncertainty measurements were similar. Consequently, only the measurement uncertainty when the robot is holding a position is illustrated in Fig. 8. The size of the points depends on the acquisition time to show the position drift over the images sequence. Positioning and orientation standard deviations are respectively $8.2 \mathrm{~nm}$ and $2.6 \mu \mathrm{rad}$.

To reduce this uncertainty, three consecutive measurements are averaged to get the platform pose. The position standard deviation drops to $6.6 \mathrm{~nm}$ taking three images into account while the angle standard deviation is only $1.85 \mu \mathrm{rad}$.

\section{Experimental Evaluation of the Precision}

The precision of the robot is quantified using repeated motions measured by the vision-based reference system. The actuators' speed is set to $20 \mu \mathrm{m} . \mathrm{s}^{-1}$, which is enough for micro- or nano- applications where high dynamic is not required. The same positions and displacements used in the simulations have been considered. Those displacements are repeated 30 times to be statistically representative. At each position, three images are taken in order to reduce the measurement noise.

Once the images are recorded, the vision processing algorithm provides the positions and orientations of the platform. Fig. 9 shows the experimental results for the first experimental case $\left(P_{1}\right)$. The maximal values of the distances to the mean for the position and orientation are respectively $40.93 \mathrm{~nm}$
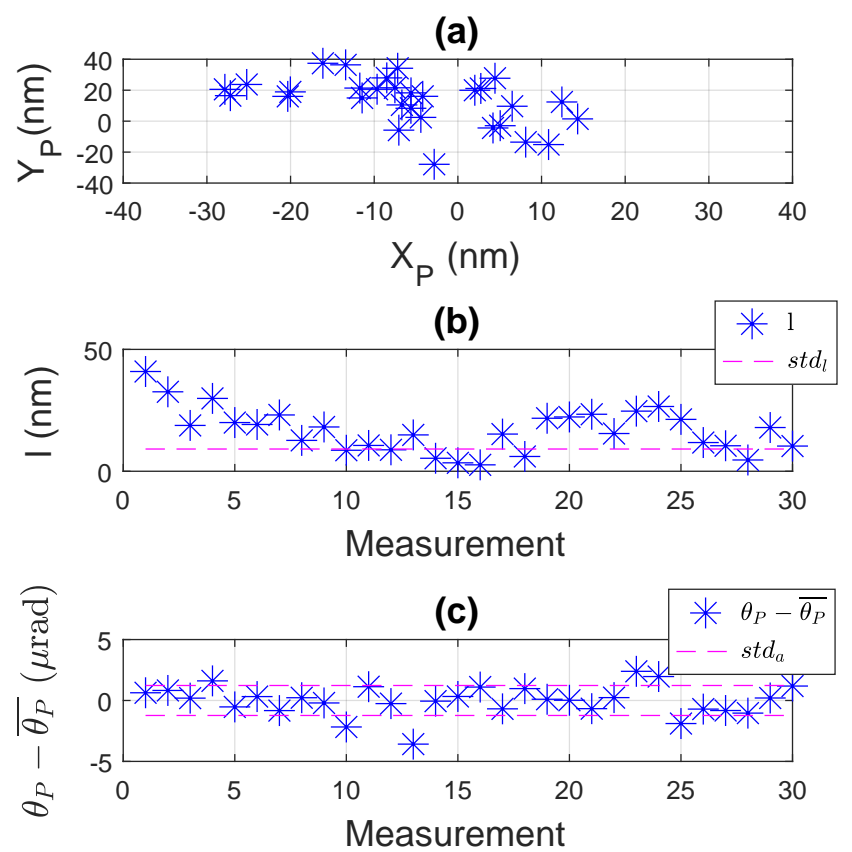

Fig. 9: (a) Platform positions during the experimental precision quantification. (b) Distances to the mean for the platform positions. (c) Differences between angles and their mean. Standard deviations $s t d_{l}, s t d_{a}$ correspond respectively to position and orientation precision.

and $4.2 \mu \mathrm{rad}$. The standard deviations, corresponding to the position and orientation precision, are $9.13 \mathrm{~nm}$ and $1.2 \mu \mathrm{rad}$. Table II shows the experimental precision results for the five cases discussed previously. One can notice that the measured deviations are extremely low, even below the estimated ones. Moreover, all precision results are at the same level as the measurement uncertainties, and we cannot conclude whether the observed deviations are mainly due to positioning errors of actuators or to environmental noise.

The precision obtained with the proposed PCR $(9.13 \mathrm{~nm}$ and $1.2 \mu \mathrm{rad}$ ) is in the same order of magnitude as classical compliant $X Y \theta$ mechanisms [6] that are specifically designed for precise applications while the workspace of the proposed PCR is significantly larger.

\section{CONCLUSION}

In this paper, we proposed a new $X Y \theta$ parallel continuum robot. The proposed structure has a safe workspace of $62.3 \mathrm{~mm}^{2}$ and $0.6452 \mathrm{rad}(39.97 \mathrm{deg})$ and is able to experimentally reach a precision of $9.13 \mathrm{~nm}$ in position and $1.2 \mu \mathrm{rad}$ in orientation. The presented results show that a planar parallel continuum robot can outperforms conventional compliant mechanisms by achieving a very high precision within a large workspace. Consequently, planar parallel continuum robots appear to be promising candidates for high-grade precision applications, which expends the potential application scope of PCRs. Future work will investigate the accuracy assessment of 
the proposed PCR and how the model's parameters influence the robot's performances.

\section{REFERENCES}

[1] C. E. Bryson and D. C. Rucker, "Toward parallel continuum manipulators," in IEEE International Conference on Robotics and Automation, May 2014, pp. 778-785.

[2] B. Brazey, R. Dahmouche, J.-A. Seon, and M. Gauthier, "Experimental validation of in-hand planar orientation and translation in microscale," Intelligent Service Robotics, vol. 9, no. 2, pp. 101-112, Apr 2016.

[3] J.-A. Seon, R. Dahmouche, and M. Gauthier, "Enhance In-Hand Dexterous Micromanipulation by Exploiting Adhesion Forces," IEEE Transactions on Robotics, vol. 34, no. 1, pp. 113-125, Feb 2018.

[4] P. R. Ouyang, R. C. Tjiptoprodjo, W. J. Zhang, and G. S. Yang, "Micromotion devices technology: The state of arts review," Int J Adv Manuf Technol, p. 17, 2008.

[5] B. Ding, Y. Li, X. Xiao, Y. Tang, and B. Li, "Design and analysis of a 3 DOF planar micromanipulation stage with large rotational displacement for micromanipulation system," Mechanical Sciences, vol. 8, no. 1, pp. 117-126, May 2017.

[6] Zeyi Wu and Qingsong Xu, "Survey on Recent Designs of Compliant Micro-/Nano-Positioning Stages," Actuators, vol. 7, no. 1, p. 5, Feb. 2018.

[7] L. Zhang and P. Yan, "Design of a parallel XY $\theta$ micro-manipulating system with large stroke," in Chinese Control and Decision Conference. IEEE, May 2016, pp. 4775-4780.

[8] D. Rus and M. T. Tolley, "Design, fabrication and control of soft robots," Nature, vol. 521, no. 7553, pp. 467-475, May 2015.

[9] D. Trivedi, C. D. Rahn, W. M. Kier, and I. D. Walker, "Soft robotics: Biological inspiration, state of the art, and future research," Applied Bionics and Biomechanics, vol. 5, no. 3, pp. 99-117, Dec. 2008.

[10] C. Ahn, X. Liang, and S. Cai, "Bioinspired Design of Light-Powered Crawling, Squeezing, and Jumping Untethered Soft Robot," Advanced Materials Technologies, p. 1900185, June 2019.

[11] J. Paik, "Soft robot design methodology for 'push-button' manufacturing," Nature Reviews Materials, vol. 3, no. 6, pp. 81-83, June 2018.

[12] J. Burgner-Kahrs, D. C. Rucker, and H. Choset, "Continuum Robots for Medical Applications: A Survey," IEEE Transactions on Robotics, vol. 31, no. 6, pp. 1261-1280, Dec. 2015

[13] W. Haouas, R. Dahmouche, J. Agnus, N. L. Fort-Piat, and G. J. Laurent, "New integrated silicon-PDMS process for compliant micromechanisms," Journal of Micromechanics and Microengineering, vol. 27 , no. 12 , p. 127001 , Oct 2017.

[14] J. Hughes, U. Culha, F. Giardina, F. Guenther, A. Rosendo, and F. Iida, "Soft Manipulators and Grippers: A Review," Frontiers in Robotics and AI, vol. 3, Nov. 2016.

[15] S. Voisembert, N. Mechbal, A. Riwan, and A. Aoussat, "Design of a Novel Long-Range Inflatable Robotic Arm: Manufacturing and Numerical Evaluation of the Joints and Actuation," Journal of Mechanisms and Robotics, vol. 5, no. 4, p. 045001, Nov. 2013.

[16] C. Girerd, K. Rabenorosoa, and P. Renaud, Combining Tube Design and Simple Kinematic Strategy for Follow-the-Leader Deployment of Concentric Tube Robots. Springer International Publishing, 2018, pp. 23-31.

[17] M. Runciman, A. Darzi, and G. P. Mylonas, "Soft Robotics in Minimally Invasive Surgery," Soft Robotics, Mar. 2019.

[18] H.-J. Kim, A. Kawamura, Y. Nishioka, and S. Kawamura, "Mechanical design and control of inflatable robotic arms for high positioning accuracy," Advanced Robotics, vol. 32, no. 2, pp. 89-104, Jan. 2018.

[19] A. L. Orekhov, C. B. Black, J. Till, S. Chung, and D. C. Rucker, "Analysis and Validation of a Teleoperated Surgical Parallel Continuum Manipulator," IEEE Robotics and Automation Letters, vol. 1, no. 2, pp. 828-835, July 2016

[20] C. B. Black, J. Till, and D. C. Rucker, "Parallel Continuum Robots: Modeling, Analysis, and Actuation-Based Force Sensing," IEEE Transactions on Robotics, vol. 34, no. 1, pp. 29-47, Feb. 2018.

[21] O. Altuzarra, D. Caballero, Q. Zhang, and F. J. Campa, "Kinematic Characteristics of Parallel Continuum Mechanisms," in Advances in Robot Kinematics. Springer International Publishing, 2019, vol. 8, pp. 293-301.

[22] Y. Sidyganov, V. Smelik, K. Semenov, D. Kostromin, and A. Medyakov, "The study of the positioning of a flexible manipulator," Journal of Applied Engineering Science, vol. 16, no. 4, p. 7, 2018.
[23] J.-P. Merlet, "Direct kinematics of planar parallel manipulators," in Proceedings of IEEE International Conference on Robotics and Automation, vol. 4, 1996, pp. 3744-3749.

[24] D. Oetomo, D. Daney, B. Shirinzadeh, and J.-P. Merlet, "Certified workspace analysis of 3RRR planar parallel flexure mechanism," in International Conference on Robotics and Automation. Pasadena, USA: IEEE, May 2008, pp. 3838-3843.

[25] P. Antunes, F. Domingues, M. Granada, and P. Andr, "Mechanical Properties of Optical Fibers," in Selected Topics on Optical Fiber Technology, M. Yasin, Ed. InTech, Feb. 2012.

[26] "Joint Committee for Guides in Metrology (JCGM) 101:2008, Supplement to the Guide to the expression of Uncertainty in Measurement (GUM)."

[27] A. N. Andre, P. Sandoz, B. Mauze, M. Jacquot, and G. J. Laurent, "Sensing one nanometer over ten centimeters: A micro-encoded target for visual in-plane position measurement," IEEE/ASME Transactions on Mechatronics, pp. 1-1, 2020.

[28] N. Tan, C. Clévy, G. J. Laurent, P. Sandoz, and N. Chaillet, "Accuracy quantification and improvement of serial micropositioning robots for in-plane motions," IEEE Transactions on robotics, vol. 31, no. 6, pp. 1497-1507, Dec. 2015.

[29] V. Guelpa, G. J. Laurent, P. Sandoz, J. Galeano Zea, and C. Clévy, "Subpixelic measurement of large 1d displacements: Principle, processing algorithms, performances and software," Sensors, vol. 14, no. 3, pp. 5056-5073, 2014

[30] V. Guelpa, P. Sandoz, M. A. Vergara, C. Clévy, N. Le Fort-Piat, and G. J. Laurent, "2D visual micro-position measurement based on intertwined twin-scale patterns," Sensors and Actuators A: Physical, vol. 248, pp. 272-280, 2016. 